\title{
Léxico y ELE: enseñanza/aprendizaje con tecnologías
}

\section{Mar Cruz Piñol ${ }^{\mathrm{i}}$}

Facultat de Filología, Universitat de Barcelona (UB) - Gran Via de les Corts Catalanes, 585. 08007 Barcelona. mcruz@ub.edu +34934035643

Este es el manuscrito del artículo que se entregó a la editorial Taylor \& Francis el 30/06/2015. Tras la aceptación por el comité editorial, la revisión por pares y la edición editorial, la versión definitiva se publicó con la siguiente referencia:

* Cruz Piñol, Mar (2015): "Léxico y ELE: enseñanza/aprendizaje con tecnologías", en Journal of Spanish Language Teaching (JSLT), 2.2, pp.

DOI: http://dx.doi.org/10.1080/23247797.2015.1105518 


\section{Léxico y ELE: enseñanza/aprendizaje con tecnologías}

En este artículo se analizará qué pueden aportar las tecnologías a la enseñanza del léxico en el marco del ELE. Se atenderá a la semántica, la morfología, la lexicografía, la terminología, la normativa, las combinaciones fijadas, etc, y en cada uno de los casos se considerarán las posibles aportaciones de los recursos de libre acceso que actualmente se encuentran en la red: diccionarios, materiales didácticos, interfaces de consulta en línea de corpus de nativos, tecnologías lingüísticas, etc. El objetivo de este artículo es doble: por una parte, presentar una revisión de los trabajos desarrollados en torno al uso de las tecnologías para la enseñanza del léxico y, así, sistematizar el camino recorrido y las vías por explorar; por otra, ofrecer unas pautas prácticas que orienten a docentes e investigadores en la incorporación de los recursos en línea para la enseñanza del léxico del ELE.

Palabras clave: léxico - enseñanza del ELE - recursos lingüísticos en línea - explotación de corpus en línea - explotación de diccionarios en línea

In this article we will analyze what new technologies can bring to the teaching of vocabulary within the SFL framework. We will take into account the different approaches to the study of vocabulary, such as semantics, morphology, lexicography, terminology, rules, and fixed combinations. In each of these cases the possible contributions of the free access online resources currently available will be considered: dictionaries, teaching materials, interfaces for the online consulting of native speaker corpus and linguistic technologies, among others. The objective of this article is twofold; on the one hand, to undertake a revision of the work done on the use of technologies for the study of vocabulary and therefore systematize the paths already taken as well as those to take, and secondly, offer practical guidance for teachers on the incorporation of online resources for vocabulary teaching in ELE.

Keywords: vocabulary - teaching of SFL - linguistic resources online- exploitation of corpus online - exploitation of dictionaries online 


\section{Introducción}

A lo largo de las próximas páginas nos ocuparemos de la potencial utilidad de diferentes tecnologías en la enseñanza/aprendizaje del léxico del ELE. Para ello atenderemos primero a la tipología de recursos (sección 2) para ver después algunos ejemplos que ilustren cómo se pueden incorporar estas tecnologías en la enseñanza/aprendizaje de contenidos concretos relacionados con el léxico del ELE (sección 3).

En todos los casos se contemplarán exclusivamente recursos accesibles de forma abierta en la red, pues consideramos esencial que todo lo que se plantee en este artículo esté al alcance de cualquier profesor que desee incorporarlo en su actividad docente. En este sentido, adelantamos que, aprovechando el medio digital en el que se presentan estas páginas, hemos utilizado hipervínculos en los ejemplos de la sección 3 , de modo que cada enlace conduce a una búsqueda que sirve para ilustrar lo que se expone en ese momento.

Nos interesa remarcar que no todos los recursos lingüísticos serán susceptibles de incorporarse en cualquier contexto didáctico, sino que la utilidad dependerá de las características de los estudiantes y de los profesores. Y es que, si bien algunas herramientas que vamos a mencionar en este artículo son muy intuitivas, otras requieren - además de una buena competencia en el uso de la lengua - una formación específica por parte de los estudiantes y de los profesores en el uso de las interfaces y (tanto o más importante) en los datos que las sostienen. A lo largo de las páginas que siguen vamos a insistir en esta necesidad de formación específica y continua, pues solo un docente bien formado podrá orientar a sus alumnos en el uso eficaz de unas tecnologías que pueden ayudar en la enseñanza/aprendizaje del léxico, pero que también pueden ser complejas, especialmente "con la llegada de las aplicaciones dentro de la web 2.0/3.0, tipo Linguee, Babla o Glosbe, donde al usuario casi se le obliga a mirar el contexto" (Buyse 2014, 105). El profesor tendrá, pues, que asumir la responsabilidad de dosificar y filtrar los diferentes recursos, sin perder de vista que "no existe relación directa entre el grado de sofisticación de la herramienta y el éxito o la rapidez del aprendizaje" (Hernández Hernández 2002, 195).

Pero no solo la formación del profesorado es crucial para el aprovechamiento eficaz de las tecnologías en la didáctica del léxico. También hábrá que tener en cuenta los diferentes niveles de competencia de los aprendices en la lengua meta, así como las habilidades de reflexión y análisis gramatical que previamente hayan adquirido en el estudio de su L1 o de otras L2. Esto nos llevará a plantearnos cuándo (en qué nivel) es conveniente introducir cada tipo de recurso, una cuestión que ha sido abordada por algunos investigadores e incluso se han publicado algunas propuestas (Blanco Carrión 2006; Buyse 2014; Oster 2009).

\section{2. ¿Qué recursos (gratuitos) útiles para la enseñanza/aprendizaje del léxico del ELE se encuentran en la red?}

\subsection{Interfaces lexicográficas en línea}

Paradójicamente, mientras sigue resultando difícil conseguir que los estudiantes de L2 integren en sus rutinas de aprendizaje la consulta de diccionarios en papel, las interfaces electrónicas ya están integradas con naturalidad en su EPA (Entorno Personal de Aprendizaje). Otra cuestión es si saben dónde, qué y cómo buscar para obtener mejores resultados, y ahí es donde la labor del docente pasa a ser fundamental. Por eso en la sección 3 llamaremos la atención sobre algunas opciones de búsqueda que permiten las interfaces en 
línea de diccionarios de aprendizaje y de diccionarios generales. Nos ocuparemos de los que han sido elaborados especialmente para la red y también de aquellos en los que, partiendo de una edición en papel, se ha diseñado una interfaz de consulta (Torruella 2002). En los de este segundo tipo, hay que tener en cuenta que, aunque cambia la interacción entre el usuario y la obra, "sus contenidos son idénticos a los de las versiones en soporte papel, e incluyen, obviamente los errores y deficiencias de aquellos" (Hernández Hernández 2002)

Queremos desde aquí llamar la atención sobre la importancia de la formación de los profesores de ELE en Lexicografía y, más concretamente, en las posibilidades que brindan las consultas expertas en las diferentes interfaces. Esta formación es fundamental para poder escoger en cada momento el diccionario en línea más adecuado a, por una parte, los contenidos léxicos que se desee tratar en el aula y, por otra, a las necesidades y los niveles de los alumnos. Insistimos en ello porque solo con la base de una buena formación continua el profesor podrá "tener claros algunos criterios para elegir un buen diccionario, como el corpus, la definición, los ejemplos, la información diatópica, distrática, diafásica, diatécnica, sinónimos y antónimos, etc.” (Nomdedeu Rull 2009, 3). Como veremos más adelante, el hallazgo del "buen diccionario" dependerá de lo que se quiera trabajar en cada momento, por lo que se recomienda conocer y combinar búsquedas avanzadas en diferentes interfaces lexicográficas.

En definitiva, partiendo del hecho de que los estudiantes de hoy se sienten cómodos con los diccionarios en soporte electrónico (Sala Caja 2015), se trata de formar al profesorado para que los pueda ayudar a mejorar su competencia como usuarios de estas interfaces y, así, contribuir a una enseñanza/aprendizaje más eficaz del léxico.

\subsection{Corpus: frecuencias y contextos}

De todos los acercamientos posibles a los corpus textuales, los listados de frecuencia son los que nos resultan menos atractivos desde una perspectiva didáctica, ya que al prescindir del contexto se pierde gran parte de la información semántica y sintáctica. Sin embargo, no se puede obviar la trascendencia de la Lexicometría y de los estudios de Disponibilidad Léxica en las decisiones más técnicas de la edición de materiales didácticos (Fernández Leyva 2015), especialmente en la línea del "control de vocabulario". No en vano el Diccionario de términos clave de ELE incluye una entrada dedicada al "vocabulario controlado", donde podemos leer que: "en la segunda mitad del siglo XX, (...) los corpus informatizados reintrodujeron la importancia de la frecuencia en la selección de vocabulario. Además, la influencia del 'movimiento de control de vocabulario' fue importante en esas décadas y lo sigue siendo al menos en dos ámbitos de la enseñanza de lenguas extranjeras: las lecturas graduadas y los diccionarios" (CVC). Con todo, aquí preferimos concentrarnos en la utilidad de las tecnologías para los docentes y no tanto para los editores de materiales, por lo que dejaremos a un lado el uso meramente estadístico de los corpus, para centrar la atención en las concordancias, más ricas en contenidos semánticos y colocacionales (Izquierdo Gil 2002).

Así pues, en la sección 3 mostraremos la utilidad de los corpus como bases de datos del léxico en contexto. Adelantamos que no nos vamos a ocupar ni de los corpus de aprendices (tan útiles en los estudios sobre adquisición derivados del análisis de la interlengua) ni de los corpus de nativos en el marco del DDL/ABD, pues ambos asuntos se abordan en otros trabajos de este mismo monográfico (Lozano, y Asención-Delaney y otros). Sí que nos referiremos a algunos corpus paralelos y multilingües, y a la web como corpus. También destacaremos la importancia de los corpus lematizados para trabajar cuestiones relacionadas con la morfología flexiva, y de los corpus de lenguajes de especialidad para abordar el léxico del español para fines específicos. Asimismo, consideraremos el concepto de "corpus" en un sentido más laxo al contemplar la utilidad de glosarios y repertorios léxicos y 
paremiológicos. En definitiva, nuestra intención es mostrar diferentes recursos léxicos disponibles en la red, para ver cómo se pueden ir combinando en distintos contextos de enseñanza (Alonso Ramos 2009; Buyse y Verlinde 2013).

\subsection{Materiales didácticos en línea}

En la sección 3 no olvidaremos los materiales didácticos para la enseñanza del léxico disponibles en línea. En esta gran sala de profesores global que es la red se pueden encontrar cientos de páginas con recopilaciones de ejercicios de ELE (pensados tanto para facilitar la tarea docente como para el autoaprendizaje), explicaciones de contenidos (en diferentes formatos multimodales, es decir, texto, imágenes, archivos sonoros, vídeos, etc.) y obras de referencia (Higueras García 2004).

Entre las obras de referencia ocupan un lugar destacado los diccionarios. Ya hemos mencionado más arriba las interfaces lexicográficas en línea, pero ahora queremos hacer especial referencia a los diccionarios que han sido confeccionados específicamente para atender las necesidades de los estudiantes de ELE. En este sentido, si bien a principios del siglo XX se constataba que "No existe un diccionario de calidad hecho específicamente para la red y adecuado para el aprendizaje de una segunda lengua" (Castillo Carballo y García Platero 2002, 18), hay que decir que hoy la situación ha cambiado gracias a proyectos como DAELE. Tal como se describe en la página de presentación, este es "un diccionario monolingüe, dirigido a estudiantes de nivel avanzado, pensado principalmente para la producción. La nomenclatura inicial se ha establecido con criterios de frecuencia y de uso, extraídos de corpus con diversos tipos de discurso. Es de consulta en línea para poder ofrecer información detallada y un número grande de ejemplos en dos o tres niveles posibles de descripción" (Battaner y Torner). Vemos, pues, que reúne tres características que lo vinculan claramente con el hilo del presente artículo: se trata de un diccionario elaborado específicamente para la enseñanza/aprendizaje del ELE, se encuentra en línea y está basado en corpus. Esta última cuestión es crucial cuando se trata de la elaboración de obras para el aprendizaje de lenguas, puesto que el corpus proporciona información muy valiosa sobre la frecuencia y el uso real de las palabras.

$\mathrm{Al}$ mencionar los diccionarios de aprendizaje es importante recordar dos cuestiones. Por una parte, que en la lexicografía didáctica es crucial conocer al usuario; y, en este sentido, en la elaboración de DAELE se ha dedicado mucha atención (mediante encuestas y pruebas con usarios) a las necesidades de los estudiantes de ELE y a su relación con la interfaz de este diccionario (Bueno 2011; Renau y Bernal 2012). La segunda cuestión que queremos traer a colación es que no es suficiente con que exista un diccionario en línea específico para el aprendizaje del ELE, sino que habrá que enseñar a los estudiantes a utilizar esta nueva herramienta (Bernal y Renau 2010; Bueno 2011; Renau y Bernal 2012); es decir, que el papel del docente (que deberá formarse para poder formar) resulta ser, una vez más, fundamental cuando se trata de incorporar las tecnologías para la enseñanza del léxico.

En relación con los ejercicios en línea para repasar y aprender cuestiones relacionadas con léxico en un entorno de autoaprendizaje, nos habría gustado atender especialmente a aquellos que se basan en corpus. Sin embargo, en el ámbito del ELE resulta todavía difícil encontrar este tipo de ejercicios (recordemos que no nos estamos refiriendo ahora al uso del corpus como input). Un ejemplo podría ser el módulo didáctico del corpus DICE, pero este se encuentra todavía en una etapa preliminar (Alonso Ramos 2013). Otro sería el prototipo VOCABLE, que, a partir de los corpus LEXESP y CLIC/TALP (y la base de datos léxica EuroWordNet), se centró en el diseño de ejercicios en torno a "las unidades léxicas relacionadas con verbos de uso básico del tipo 'dar' pues son esenciales en el uso de la lengua, son polisémicos y presentan un alto grado de idiomaticidad" (Morante $2004 \mathrm{~s} / \mathrm{p}$ ); pero este 
prototipo no está accesible en la red. Así pues, mencionaremos otros ejercicios en línea de vocabulario, aunque no estén basados en corpus.

\subsection{Tecnologías lingüísticas de acceso libre}

También en la sección siguiente se verán algunas posibilidades que para la enseñanza/aprendizaje del léxico ofrecen las tecnologías diseñadas en el marco del procesamiento del lenguaje natural (PLN). Una vez más, se verá que algunas son sencillas mientras que otras requieren que los profesores se instruyan para poderlas utilizar con fines didácticos.

\section{3. ¿Qué se se puede enseñar/aprender del léxico del ELE con la ayuda de las}

\section{tecnologías?}

\subsection{Cuestiones relacionadas con la onomasiología y la semántica}

Señalar una cosa para averiguar su nombre es la necesidad más básica de comunicación, y ese camino que va de la idea (la cosa, concepto o significado) a la palabra (la forma o el significante) — es decir, la Onomasiología - se puede ilustrar en la red, dado su carácter esencialmente multimedia. No en vano ya desde que se empezó a utilizar Internet para la enseñanza/aprendizaje de lenguas, proliferaron los ejercicios en línea de vocabulario en los que cada palabra iba acompañada de "su imagen", en la línea de una metodología de larga tradición (recordemos el Orbis sensualium pictus de Comenius). No nos vamos a detener aquí en esta cuestión porque ya la hemos abordado en un estudio anterior (Cruz Piñol 2002) y preferimos dedicar estas páginas a otros recursos, quizá menos habituales en las aulas de ELE. El lector que desee consultar ejercicios interactivos de vocabulario (muchos de ellos con imágenes) puede recurrir al enlace que se ofrece en el Anexo final.

El camino inverso, el que va de la palabra al significado, nos conduce a los diccionarios. Sin perder de vista el hilo conductor de este artículo, iremos viendo algunas diferencias entre las principales interfaces de consulta lexicográfica disponibles de forma gratuita en la red, para ayudar al profesor de ELE a conocer diferentes opciones y escoger en cada situación la que se ajuste mejor a sus necesidades. Con todo, debemos recordar que las búsquedas avanzadas en los diccionarios en línea y las posibilidades que presentan en el aula de ELE es aquí solo una pequeña parte de las tecnologías que queremos mostrar, por lo que no podremos extendernos en ello, aunque contamos con dedicarle más atención en el futuro.

Por lo que se refiere al DRAE, la interfaz de consulta que ofrece la RAE permite acceder a la misma información que contiene el diccionario en papel (Hernández Hernández 2002), con la ventaja (más allá del acceso abierto, cuestión nada desdeñable) de que va incorporando las enmiendas y adiciones aprobadas por los académicos. Por otra parte, la consulta en línea del DRAE incorpora un conjugador verbal y permite la búsqueda por formas flexionadas, dos cuestiones a las que nos referiremos más adelante. Pero existen otras interfaces de acceso al DRAE (como GoodRae y DiRae) que, como enseguida veremos, aprovechan mejor las posibilidades de la hipertextualidad para la consulta de la base de datos que es un diccionario.

Más allá del diccionario académico, sin adentrarnos en las diferencias entre diccionarios normativos y de uso, y con la atención siempre puesta en las interfaces en línea, hay que tener en cuenta otros enriquecimientos que puede ayudar en la comprensión del 
léxico: por ejemplo, que el diccionario incluya los sinónimos y antónimos de las diferentes acepciones, como Salamanca, WordReference (Espasa) y Diccionarios.com (Larousse); o que describa las diferentes estructuras argumentales de cada unidad o acepción (como Salamanca y DAELE). Además, todos estos diccionarios incluyen ejemplos de las palabras en contexto, algunos tomados de corpus, como DAELE o WordReference (Espasa), y este ofrece además la traducción al inglés y al francés.

También pueden ser de utilidad las interfaces que informan sobre las clases aspectuales, sobre los roles semánticos y sobre redes y marcos semánticos, como las que se elaboran desde el PLN, como Grial/SenSem, AnCora, Adesse, MCR, SpanishFrameNet o MultiWordNet, este último, además, con la traducción a otras lenguas. Sin embargo, para el uso de estas interfaces, como decíamos más arriba, serán necesarias unas ciertas competencias tanto por parte de los estudiantes como de los docentes. Por ejemplo, Blanco Carrión (2006) ha sugerido unos prerequisitos para el uso didáctico de FrameNet: que los estudiantes sean adultos, que tengan conciencia del concepto de redes semánticas (y sepan utilizarlas al menos en su propia lengua) y que tengan capacidad analítica. Pero si estas aplicaciones resultan demasiado complejas para los alumnos (o los profesores), hay tecnologías más simples que ayudan a tomar conciencia de las redes semánticas que se establecen entre las palabras de una lengua: algunas interfaces de consulta del DRAE - como DiRae y GoodRAE - permiten la búsqueda de palabras contenidas en la definición y así, por ejemplo, es posible ver todas las palabras del DRAE referidas a "plantas" (los hipónimos y su hiperónimo).

No podemos terminar este apartado referido a cuestiones semánticas sin considerar la utilidad de los corpus paralelos, que recogen textos traducidos, generalmente con las versiones alineadas, de manera que se puede buscar una palabra y leer simultáneamente sus apariciones en textos en diferentes lenguas. No nos interesa aquí discutir sobre la utilidad de la traducción en el aprendizaje de lenguas segundas, sino llamar la atención sobre la existencia de corpus paralelos accesibles de forma gratuita en la red. Es el caso del proyecto OPUS, que permite ver en paralelo traducciones de textos en decenas de lenguas, o del famoso Linguee, que junto a la traducción de palabras (y combinaciones) muestras ejemplos reales de su uso en contextos tomados de la red (Alonso Jiménez 2013). La utilidad de Linguee en el aprendizaje del léxico ha sido observada en algunos estudios basados en la experiencia del aula (Buyse y Verlinde 2013; Buyse 2014) y la conclusión es que el uso de esta herramienta favorece la expresión escrita, especialmente si se combina con otros recursos, a partir de un nivel A2.

\subsection{Cuestiones relacionadas con las combinaciones frecuentes de palabras}

Adentrarse en el terreno de las coapariciones de palabras resulta aterrador si se pretende un consenso terminológico, pero fascinante si se centra la atención en el concepto. Quizá por eso, "en los manuales de ELE las coapariciones son agrupadas - pese a su heterogeneidad- en un mismo concepto, al que se alude con diferentes denominaciones" (Sánchez Rufat 2015), una solución práctica que nos parece la más didáctica. Para tomar conciencia de las unidades multipalabra podemos recurrir a muchas bases de datos en línea, empezando por los diccionarios DICE o ScieLex (Verdaguer et al. 2010), y sin olvidar los repertorios paremiológicos como Badare (Gargallo Gil 2012).

En la línea del uso de la web como corpus, es ya una rutina para muchos estudiantes (y profesores) teclear una palabra en Google para saber sobre ella. Si lo hacemos, por ejemplo, con rendir, veremos que automáticamente nos sugiere esa palabra seguida de cuentas, pleitesía o tributo, y algo parecido ocurre con GoogleTranslator. En ambos casos estamos, quizá sin saberlo, utilizando un corpus que nos informa sobre las combinaciones más frecuentes de una palabra. Asimismo, en los corpus de nativos es posible consultar (bajo 
diferentes denominaciones), qué palabras acompañan a la que nos interesa, por ejemplo, CREA ("agrupaciones"), CORPES ("proximidad") o el Corpus del español ("colocados"). Y la unión entre corpus y diccionario la podemos ver ejemplificada en la interfaz DiRae, que muestra, junto a la definición (del DRAE), las palabras que suelen aparecer inmediatamente a la derecha o a la izquierda del lema consultado.

También WortSchatz localiza en un corpus (Leipzig Corpora) las palabras que con frecuencia aparecen en el entorno de una unidad léxica, inmediatamente a su derecha, inmediatamente a su izquierda, y en cualquier lugar en la misma frase. Los resultados se ofrecen en forma de listado y también gráficamente, en una especie de telaraña léxica que se puede ver más o menos tupida (según si se escoje la presentación con más o menos palabras). En la línea de lo que venimos advirtiendo sobre el cuidado que hay que tener al incorporar recursos de este tipo en las aulas, algunos autores han sugerido que WortSchatz sería útil con estudiantes de nivel B1 o superior (Buyse 2014; Oster 2009).

Por último, y sin adentrarnos en el uso de los corpus de nativos desde la perspectiva del ABD, nos gustaría solo mencionar algunas experiencias desarrollada en el contexto universitario de enseñanza de filologías extranjeras, en las que se han empleado corpus para favorecer la conciencia de los estudiantes sobre la importancia de las colocaciones y para facilitar su aprendizaje, tanto en entornos docentes virtuales (Pérez Basanta 2006; Moreno Jaén 2009) como presenciales (Laso y Comelles 2012; Sevilla Muñoz 2012). En todos los casos, a partir de estudios basados en la experiencia docente, se constata la utilidad de las bases de datos de muestras reales de la L2 en contexto. Hemos querido destacar estas situaciones docentes en las que a los estudiantes se les supone un considerable nivel de reflexión lingüística para recordar, una vez más, que la utilidad de unas u otras tecnologías debe mesurarse en función de las competencias de los alumnos.

\subsection{Cuestiones relacionadas con la morfología}

\subsubsection{Sobre la flexión y la derivación}

Existen en la red numerosas aplicaciones que muestran el paradigma flexivo del verbo, con ciertas diferencias que indicamos a continuación, para ayudar al docente a escoger entre una $u$ otra según qué cuestiones referidas a la flexión verbal desee trabajar. Así, por ejemplo, el DRAE en línea ofrece en las entradas verbales un enlace a la conjugación, sin olvidar las diferentes denominaciones que reciben los tiempos; WordReference ofrece un paradigma más completo, en el que resalta gráficamente los tiempos desusados, y da información sobre los tipos de irregularidades y los modelo de conjugación; también Onoma y ULPGC-TIP muestran el tipo de conjugación y las irregularidades y el último, además, permite conocer la frecuencia de cada forma flexionada en el corpus CREA. Además, algunos de estos conjugadores, como veremos más adelante, contemplan la variación diatópica del español.

La flexión de nombres y adjetivos también se puede ampliar llevando al aula aplicaciones elaboradas por equipos que trabajan en el PLN, como los flexionadores del CLIC y del GEDLC-ULPGC. Las demos del segundo muestran las posibilidades flexivas de género y número de unidades nominales (y ahí es capaz de reconocer, por ejemplo, las palabras sin cambio formal de género, como "estudiante", y los heterónimos, como "hombre"/“mujer") y también de otras clases de palabras.

En relación con los afijos, algunas interfaces lexicográficas (como Clave, y DiRae y GoodRae para el DRAE) permiten buscar, por ejemplo, todas las palabras lexicalizadas con el sufijo *ito, todos los adverbios en *mente o todos los parasintéticos formados con en*ecer. Más allá de los diccionarios, el GEDLC-ULPGC ofrece una aplicación que muestra las 
posiblidades combinatorias de una palabra con sufijos apreciativos (diminutivos, aumentativos y peyorativos) y con hasta tres prefijos.

\subsubsection{Lemas y formas}

En las lenguas flexivas, el reconocimiento del lema es imprescindible, sin ir más lejos, para poder buscar una palabra en un diccionario. Hoy en día, las interfaces en línea de la mayoría de los diccionarios permiten la búsqueda a partir de las formas flexionadas, es decir, que si se busca fue indica que puede corresponder a uno de estos dos lemas: ser o ir. Es el caso, por ejemplo, de WordReference (Espasa), GoodRae (DRAE) y de la propia interfaz del DRAE en la web de la RAE.

Más allá de los diccionarios, la distinción entre formas y lemas es básica también cuando se manejan corpus textuales. Ya hemos explicado en un trabajo anterior (Cruz Piñol 2012) que los corpus lematizados son útiles para la enseñanza/aprendizaje del ELE en la medida en que permiten observar en sus contextos las formas flexionadas de un lema y, de este modo, se convierten en una herramienta de ayuda para la asimilación inductiva del paradigma flexivo. En los corpus lematizados (como el Corpus del español, CORPES, AnCora y otros que se pueden consultar desde el Anexo) se pueden buscar ocurrencias de un lema como casa y, precisamente porque la información que se ha introducido previamente en la base de datos ha discriminado el verbo (lema casar) del nombre (lema casa), se obtendrán exclusivamente los contextos en los que aparece el segundo. E incluso se puede llegar a un grado más de precisión, posible porque estos corpus disponen también de información referente a la categoría gramatical (es decir, además de indicar los lemas de cada una de las unidades léxicas en su contexto real de aparición en el corpus, se ha indicado cuál es su categoría gramatical): de este modo, es posible localizar las ocurrencias de un lema con una determinada categoría, por ejemplo, solo los casos correspondientes al lema ser como verbo. El nivel de análisis morfológico en estos corpus es elevado, lo cual, por una parte, es sumamente útil desde el punto de vista linguiístico y, por otra, es una complejidad que hace pensar que serán útiles con estudiantes de un nivel B2 o superior (Buyse 2014).

Hay que mencionar también las aplicaciones fruto de la investigación en el PLN que muestran los posibles lemas de una forma flexionada, con diferentes grados de detalle: algunas indican el o los lemas posibles de una palabra sin tener en cuenta el contexto (como el lematizador de Signum), otros dan además información morfológica (categoría gramatical, género, número, tiempo verbal, etc., como el de FreeLing, el del Clic o el de SpanishFrameNet) y algunos incluso son capaces de reconocer los lemas de unidades pluriverbales (como el lematizador del GEDLC-ULPGC o el diccionario de SpanishFrameNet). Los hay también que son capaces de mostrar el lema que corresponde a la unidad léxica en un contexto determinado, es decir, al introducir un enunciado es capaz de discriminar, por ejemplo, cuándo el lema de fue es ser y cuándo ir (como el de FreeLing o el del $\underline{\text { Clic). }}$. E incluso los hay que muestran qué relaciones sintácticas se establecen entre las unidades que aparecen en una frase (como el de FreeLing o el del Clic), pero en este artículo nos estamos centrando en el léxico y el análisis sintáctico se escapa de nuestros objetivos.

\subsection{Cuestiones relacionadas con la terminología}

Un diccionario general puede constituir una fuente de información sobre la terminología de un lenguaje específico si la interfaz permite la consulta por marcas lexicográficas. Por ejemplo, con DiRae o GoodRae se pueden buscar todas las palabras con la marca Econ. en alguna de sus acepciones y, así, obtener un "glosario" de terminología económica a partir de la información contenida en el DRAE. De este modo, se comprueba que "Otra virtud del 
diccionario en formato electrónico es que puede ser al mismo tiempo un diccionario general y muchos diccionarios particulares, tanto por el tipo (sinonímico, etimológico, dialectal, etc.) como por el nivel de las informaciones que da (acepciones principales, acepciones terminológicas, subentradas compuestas, etc.)" (Torruella 2002, 23).

También en algunos corpus generales es posible restringir la búsqueda de apariciones de una determinada palabra a textos de una temática específica, como ocurre con los corpus de referencia CREA y CORPES. Pero al referirnos a la terminología son especialmente relevantes los corpus multilingües, que -como Grial o el corpus técnico del IULA-reúnen exclusivamente textos de lenguajes de especialidad, y los diccionarios bilingües que -como Linguee- conceden una especial atención a este tipo de textos. Asimismo, hay que mencionar los numerosísimos glosarios de terminología disponibles en la red (véase el Anexo) y los foros sobre terminología de WordReference.

\subsection{Cuestiones relacionadas con la sociolingüística}

Por lo que se refiere a la dialectología, como hemos indicado más arriba, algunas aplicaciones tienen en cuenta las variedades diatópicas al presentar el paradigma verbal, como por ejemplo el DRAE y el de ULPGC-TIP, que contemplan la distinción tú/vos y vosotros/ustedes.

Otro recurso que se puede utilizar para introducir cuestiones sociolingüísticas en el aula son las nubes de palabras, unas aplicaciones que presentan de forma visual las palabras de un texto (generalmente solo las unidades léxicas, es decir, tras descartar los elementos gramaticales, como artículos y preposiciones, que siempre son los más frecuentes), en una gradación de colores y tamaños que pone de relieve la frecuencia léxica. Existen numerosos programas gratuitos para diseñar estas "nubes" que dibujan el léxico de un texto y, a través de él, su contenido. Un ejemplo de aplicación de este recurso es el artículo que se publicó en la versión digital de La Vanguardia al finalizar el 2013, bajo el título "¿De qué han hablado los lectores en sus cartas durante el 2013?”, en el que Mónica Cebrián ilustró su artículo con las nubes de palabras obtenidas (con Wordle) a partir de las cartas de los lectores.

\subsection{Cuestiones relacionadas con la norma y el uso}

Queremos terminar esta sección mencionando la utilidad de las tecnologías en casos de dudas léxicas. Al plantear esta cuestión es probable que lo primero que venga a la mente sean los correctores automáticos y los diccionarios de dudas. Respecto a los primeros, solo recordaremos que constituyen una ayuda en la medida en que revisan, pero que hay muchas cuestiones que no son capaces de corregir (García Asensio 2014), y respecto a los segundos, remitiremos a los enlaces que se ofrecen en el Anexo. Preferimos dedicar este espacio a otras cuestiones, quizá menos conocidas.

Existen algunos recursos lexicográficos que, junto al lema, informan de si plantea alguna dificultad entre los nativos. Por ejemplo, la interfaz DiRae señala cuándo una palabra tiene una entrada en el Diccionario Panhispánico de Dudas. Por su parte, WordReference vincula las palabras con los foros en las que han sido mencionadas, tanto en contextos de uso general (enlaza con la web como corpus) como en casos de metalenguaje (cuando han sido tema de consulta en los foros de dudas de este portal). Por otra parte, y puesto que la acentuación está estrechamente relacionada con la división en sílabas, al trabajar esta cuestión normativa puede resultar útil saber que algunas interfaces indican el número de sílabas que tiene la palabra, como Clave y DiRae (la última, además, dice si la palabra es aguda, llana o esdrújula).

También es pertinente recordar la utilidad de los corpus como "juez" en casos de dudas o discusiones relacionadas con el léxico (Alonso Pérez-Ávila 2007; Humblé 2001; Sala 
Caja 2004a, 2004b, 2009). A este respecto, estamos de acuerdo con Kris Buyse (en Cruz Piñol et al. 2012) en que las consultas de concordancias en corpus fomentan la autonomía del aprendizaje, especialmente ante las dudas léxicas, pues permiten ver, a partir de muestras reales de la lengua en uso, cuáles son las expresiones más frecuentes producidas por hablantes nativos. En este sentido, recordemos que la web es en sí misma un gran corpus con el que los estudiantes están bien familiarizados, así que se puede utilizar como primer paso para introducir después en el aula otros corpus más controlados.

\section{Conclusión}

Tras lo expuesto en las páginas precedentes se puede concluir que existen actualmente en la red diferentes tecnologías que pueden ser útiles para la enseñanza y el aprendizaje del léxico del ELE, aunque no hayan sido diseñadas especialmente con fines didácticos. Hemos querido hacer especial hincapié en los distintos grados de complejidad de estos recursos porque de ahí se deriva otra cuestión que nos parece fundamental: la formación de los profesores para ser capaces de calibrar qué se puede obtener de cada tecnología y cuándo conviene introducírla en el aula. Y es precisamente el contacto con las experiencias docentes lo que nos ha ayudado a mostrar que también el nivel de los alumnos determinará si es o no adecuado incorporar una u otra tecnología. En definitiva, sobre la base de la formación continua, abogamos por un mayor aprovechamiento de las tecnologías que se nos brindan en la red, pues estamos convencidos de que tienen mucho que aportar en la enseñanza/aprendizaje del léxico.

\section{Anexo: selección de enlaces}

A continuación ofrecemos una selección de enlaces útiles para ampliar lo expuesto en este artículo:

- Diccionarios en línea: http://delicious.com/marcruzpinol/diccionarios

- Colocaciones: http://delicious com/marcruzpinol/colocaciones

- Paremiología: http://delicious.com/marcruzpinol/paremiologia

- Sinónimos/antónimos: http://delicious.com/marcruzpinol/sinonimia

- Frecuencias léxicas: http://delicious.com/marcruzpinol/frecuencias_lexicas

- Terminología: http://delicious.com/marcruzpinol/terminologia

- Nubes de palabras: http://delicious.com/marcruzpinol/nubes_de_palabras

- Corpus de aprendices: http://delicious.com/marcruzpinol/corpus,de_aprendices

- Corpus paralelos/multilingües: http://delicious.com/marcruzpinol/corpus,paralelos_y_multilingues

- Corpus lematizados: http://delicious.com/marcruzpinol/corpus,lematizado

- Web como corpus: http://delicious.com/marcruzpinol/web_como_corpus

- Más corpus: http://delicious.com/marcruzpinol/corpus

- Materiales didácticos: http://delicious.com/marcruzpinol/Propuestas_de_clases_Materiales_didacticos

- Ejercicios de vocabulario: http://delicious.com/marcruzpinol/ejercicios,lexico

- Normativa: http://delicious.com/marcruzpinol/normativa

- Sociolingüística: http://delicious.com/marcruzpinol/aplicaciones_sociolinguistica 


\section{Bibliografía}

Alonso Jiménez, E. 2013. "Linguee y las nuevas formas de traducir". Skopos: revista internacional de traducción e interpretación 2: 5-28.

http://rio.upo.es/xmlui/bitstream/handle/10433/851/2013_Alonso_Linguee_Skopos.pdf?s equence $=1$

Alonso Pérez-Ávila, E. 2007. "El corpus lingüístico en la didáctica del léxico del ELE". Boletín de ASELE 37: 11-25. http://formespa.rediris.es/pdfs/asele37.pdf

Alonso Ramos, M. 2009. "Hacia un nuevo recurso léxico: ¿fusión entre corpus y diccionario?”. En A survey of corpus-based research, eds. P. Cantos Gómez y A. Sánchez Pérez, 1191-207. Murcia: Aelinco. http://www.um.es/lacell/aelinco/contenido/pdf/82.pdf

Alonso Ramos, M. 2013. "Colocaciones, diccionario y corpus de aprendices". En Eugenio Coseriu, in memoriam, XIV Jornadas de Lingüística, eds. M. Casas Gómez y R. Vela Sánchez, 57-71. Universidad de Cádiz. http://www.grupolys.org/biblioteca/Alo2013a.pdf

Alvar Ezquerra, M. 2004. "La frecuencia léxica y su utilidad en la enseñanza del español como lengua extranjera". En Las Gramáticas y los diccionarios en la enseñanza del español como segunda lengua: deseo y realidad, ed. M.A. Castillo, 19-39. Sevilla: ASELE - Universidad de Sevilla. http://cvc.cervantes.es/ENSENANZA/biblioteca_ele/asele/pdf/15/15_0017.pdf

Arrarte, G., y J. Llisterri. 1997. "Industrias de la lengua y enseñanza del español como lengua extranjera". Carabela 42: 27-38.

Battaner, P., y S. Torner (Dir.). DAELE - Diccionario de Aprendizaje del español como lengua extranjera (Presentación). http://www.iula.upf.edu/rec/daele/ (01/06/2015).

Bernal, E., e I. Renau. 2010. “¿Lo que necesitan es lo que encuentran? Reflexiones a propósito de la representación de los verbos en los diccionarios de aprendizaje de español". En Proceedings of the XIV Euralex International Congress, eds. A. Dykstra y T. Schoonheim, 484-96. Ljouwert: Fryske Akademy. http://www.euralex.org/elx_proceedings/Euralex2010/032_Euralex_2010_2_BERNAL RENAU_Q_Lo que necesitan es lo que encuentran_Q Reflexiones a proposito de la representacion d.pdf

Blanco Carrión, O. 2006. "Framenet as a Corpus Tool for the Learning of Second Languages and for the Lexical Awareness of One's First Language". Porta Linguarum. Revista Internacional de Didáctica de las Lenguas Extranjeras 6: 67-76. http://www.ugr.es/ portalin/articulos/PL_numero6/blanco.pdf

Bueno, R. 2011. "El Diccionario de aprendizaje de español como lengua extranjera (DAELE): una nueva herramienta en el aprendizaje de LE". Domínios de Lingu@gem 5(2): 202-16. http://www.seer.ufu.br/index.php/dominiosdelinguagem/article/view/13742

Buyse, K. 2014. "Una hoja de ruta para integrar las TIC en el desarrollo de la expresión escrita: recursos y resultados". Journal of Spanish Language Teaching 1(1): 101-15. http://www.tandfonline.com/doi/pdf/10.1080/23247797.2014.898516

Buyse, K., y S. Verlinde. 2013. "Possible Effects of Free on Line Data Driven Lexicographic Instruments on Foreign Language Learning: The Case of Linguee and the Interactive Language Toolbox". Procedia - Social and Behavioral Sciences 95: 507-12. http://www.sciencedirect.com/science/article/pii/S1877042813041955

Calvi, M.V., y F. San Vicente. 2003. Didáctica del léxico y nuevas tecnologías. ViareggioLucca: Mauro Baroni - IC de Milán - Universidad de Bolonia.

Castillo Carballo, M.A., y J.M. García Platero. 2002. "Internet y enseñanza de vocabulario". En Tecnologías de la información y de las comunicaciones en la enseñanza de la E/LE, 
ed. A. Gimeno, 17-26. Valencia: UP de Valencia - Asele.

http://cvc.cervantes.es/ensenanza/biblioteca_ele/asele/pdf/12/12_0017.pdf

Criado Sánchez, R., y A. Sánchez Pérez. 2009. "Vocabulary in EFL Textbooks: A Contrastive Analysis against Three Corpus-Based Word Ranges". En A Survey of Corpus-Based Research, eds. P. Cantos Gómez y A. Sánchez Pérez, 862-75. Murcia: Aelinco. http://www.um.es/lacell/aelinco/contenido/pdf/58.pdf

Cruz Piñol, M. 2002. Enseñar español en la era de Internet: la WWW y la enseñanza del español como lengua extranjera. Barcelona: Octaedro.

Cruz Piñol, M. 2012. Lingüística de corpus y enseñanza del español como 2/L. Madrid: Arco Libros.

Cruz Piñol, M., K. Buyse, V. González Argüello y N. Tukahara. 2012. “¿Qué queremos de la red y para qué? Nuevas perspectivas en el uso de la red en la enseñanza del ELE”. En La red y sus aplicaciones en la enseñanza-aprendizaje del español L/E, eds. C. Hernández González, A. Carrasco Santana, y E. Álvarez Ramos, 31-59. Valladolid: Ediciones de la Universidad.

CVC. "Vocabulario Controlado". Diccionario de términos clave de enseñanza de español como lengua extranjera.

http://cvc.cervantes.es/ensenanza/biblioteca_ele/diccio_ele/diccionario/vocabulariocontr olado.htm $(17 / 12 / 2014)$

Fernández Leyva, H. 2015. "Posible aplicación del índice de disponibilidad léxica a la selección del vocabulario de manuales de ELE". MarcoELE 20 http://marcoele.com/descargas/20/fernandez-disponiblidad_lexica.pdf

García Asensio, M.Á. 2014. "Ortografía: lo que el corrector automático de textos no sabe corregir”. En Manual de escritura académica y profesional, ed. E. Montolío, 45-70. Barcelona: Ariel.

Gargallo Gil, J.E. 2012. “BADARE, una herramienta multidisciplinar”. En Unidades Fraseológicas y TIC, ed. M.I. González Rey. CVC. http://cvc.cervantes.es/lengua/biblioteca_fraseologica/n2_gonzalez/gargallo.htm

Hernández Hernández, H. 2002. "El diccionario informatizado como recurso en la enseñanza de E/LE: límites y posibilidades". En Tecnologías de la información y de las comunicaciones en la enseñanza de la E/LE, ed. A. Gimeno, 191-200. Valencia: UP de Valencia - Asele. http://cvc.cervantes.es/ensenanza/biblioteca_ele/asele/pdf/12/12_0191.pdf

Higueras García, M. 2004. “Internet en la enseñanza del español”. En Vademécum para la formación de profesores. Madrid: SGEL, 1061-85.

Humblé, P. 2001. "O uso de corpora no ensino de línguas. Alguns exemplos do português e do espanhol". En Lingüística e ensino: novas tecnologias, eds. L. Grimm Cabral, y P. de Souza, 157-80. Blumenau: Nova Letra.

Izquierdo Gil, M.C. 2002. "Los córpora electrónicos en la enseñanza del vocabulario español: la utilidad pedagógica de las concordancias". En Tecnologías de la información y de las comunicaciones en la enseñanza de la E/LE, ed. A. Gimeno, 201-12. Valencia: UP de Valencia - Asele.

http://cvc.cervantes.es/ensenanza/biblioteca_ele/asele/pdf/12/12_0191.pdf

Laso, N.J., y E. Comelles. 2012. "Usos metodológicos de corpus textuales en el aula de lexicología y morfología”. En Experiencias de innovación docente en la enseñanza universitaria de las humanidades, eds. M. Trenchs Parera y M. Cruz Piñol, 55-72. Barcelona: Octaedro.

Morante, R. 2004. "VOCABLE: una plataforma para el aprendizaje de vocabulario asistido por ordenador". RedELE 2. http://www.mecd.gob.es/dctm/redele/Material- 
$\underline{\text { RedEle/Revista/2004_02/2004_redELE_2_12Morante.pdf?documentId=0901e72b80e06 }}$ $73 \mathrm{e}$

Moreno Jaén, M. 2009. "Recopilación, desarrollo pedagógico y evaluación de un banco de colocaciones frecuentes de la lengua inglesa a través de la lingüística de corpus y computacional." Tesis, Universidad de Granada. http://digibug.ugr.es/handle/10481/2311

Nomdedeu Rull, A. 2009. "Diccionarios en Internet para el aula de ELE”. RedELE 15. http://www.mecd.gob.es/dctm/redele/Material-

$\underline{\text { RedEle/Revista/2009_15/2009_redELE_15_02Nomdedeu.pdf?documentId=0901e72b80 }}$ dd 8826

Oster, U. 2009. "La adquisición de vocabulario en una lengua extranjera: de la teoría a la aplicación didáctica". Porta Linguarum 11: 33-50.

Pérez Basanta, C. 2006. Fundamentos teóricos y prácticos de ADELEX: una investigación sobre la evaluación y el desarrollo de la competencia léxica a través de las nuevas tecnologías. Granada: Comares.

Renau, I., y E. Bernal. 2012. "El diccionario de aprendizaje de español como lengua extranjera (DAELE): primera prueba de usuarios". En Avances de Lexicografía Hispánica: II, eds. A. Nomdedeu, E. Forgas, y M. Bargalló, 245-59. Tarragona: Universitat Rovira i Virgili.

Sala Caja, L. 2004a. "Aliarse con Internet para aprender (sobre el) vocabulario". Carabela 56: 121-43.

Sala Caja, L. 2004b. "Traspasar los limites del diccionario". The Journal of the Faculty of Foreign Studies, Aichi Prefectural University. Language and literature 36: 251-75. http://ci.nii.ac.jp/naid/110004029465/en/

Sala Caja, L. 2009. "Las TIC en el aula de espanol como lengua extranjera: videos digitales y YouTube". Journal of international relations and comparative culture 7(2): 239-49. http://ci.nii.ac.jp/naid/110007163882/en/

Sala Caja, L. 2015. "Actitudes de estudiantes y profesores hacia los diccionarios electrónicos portátiles”. En La Enseñanza de ELE centrada en el alumno. Getafe: Universidad Carlos III-Asele.

Sánchez Rufat, A. 2015. "El verbo 'dar' en el español escrito de aprendientes de L1 inglés: estudio comparativo entre hablantes no nativos y hablantes nativos basado en corpus". Tesis, Universidad de Cáceres.

Sevilla Muñoz, M. 2012. "Utilización de recursos en línea en la enseñanza/aprendizaje de traducción de unidades fraseológicas". En Unidades Fraseológicas y TIC, ed. M.I. González Rey. CVC.

http://cvc.cervantes.es/lengua/biblioteca_fraseologica/n2_gonzalez/m_sevilla.htm

Torruella, J. 2002. "Los diccionarios en la red”. En Filología en Internet, 21-41. Bellaterra: $\mathrm{UAB}$.

Verdaguer, I., E. Comelles, N.J. Laso, E. Giménez, y Danica Salazar. 2010. "SciE-Lex: An Electronic Lexical Database for the Spanish Medical Community". En E-Lexicography in the 21st Century: New Challenges, New Applications, eds. S. Granger, y M. Paquot, 325-34. Louvain-la-Neuve: UCL.

${ }^{\mathrm{i}}$ Este artículo se inscribe en el equipo GReLiC (Grup de recerca en Lexicologia i Lingüística de corpus), grupo consolidado por la Generalitat de Catalunya, SGR 2014-2016 (http://www.ub.edu/grelic/). 
${ }^{i i}$ La bibliografía en torno al uso de la estadística para elaborar obras lexicográficas didácticas es muy extensa, pero ya hemos apuntado más arriba que esta es una cuestión de la que no nos podemos ocupar en este trabajo. Nos limitaremos aquí a citar, a modo de apoyo y sin perder de referencia el ELE, dos contribuciones en las que se insisten en ello: una conferencia plenaria pronunciada en un congreso de ASELE (Alvar Ezquerra 2004), y un artículo en coautoría con el responsable del corpus Cumbre (Criado Sánchez y Sánchez Pérez 2009).

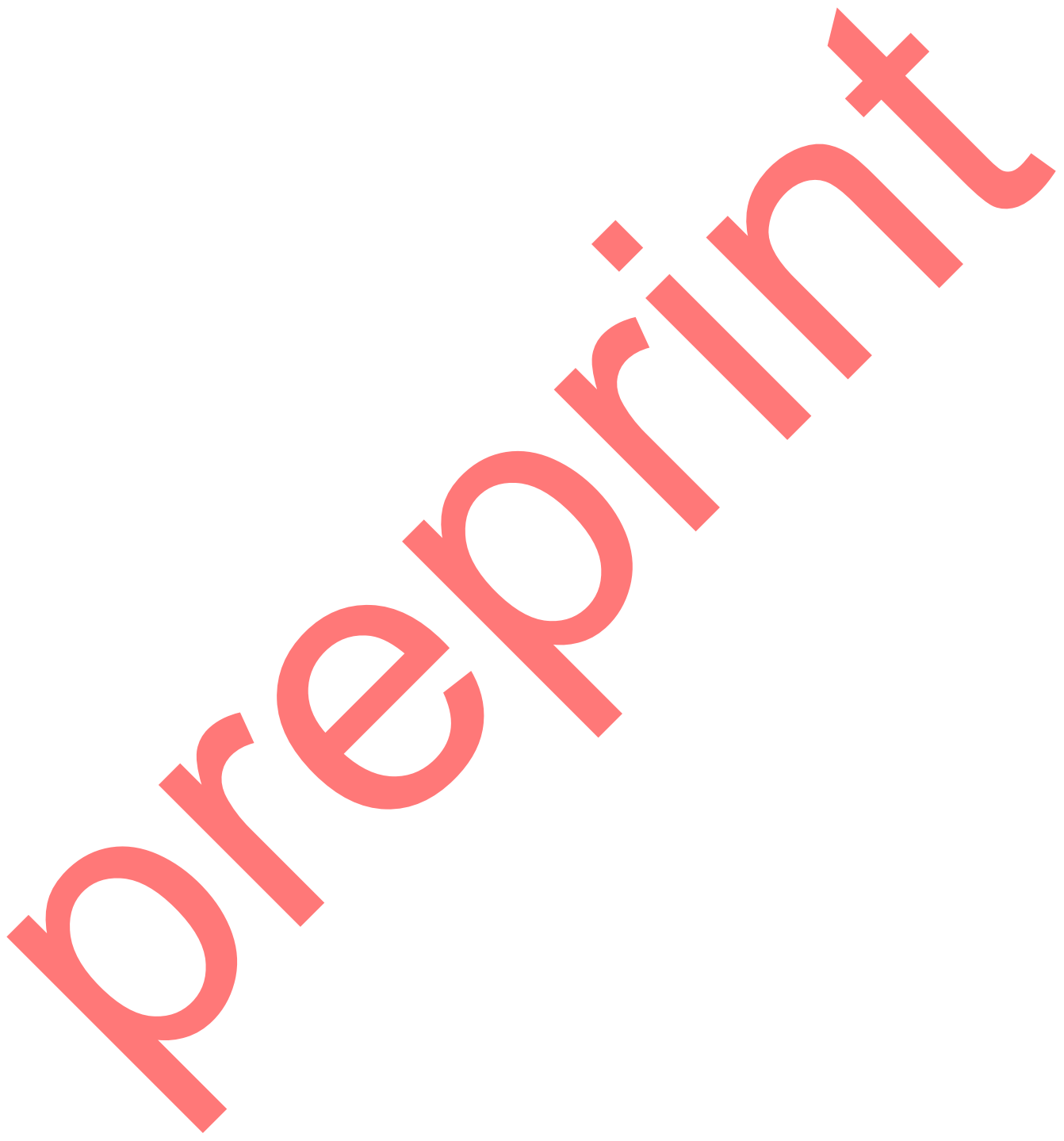

